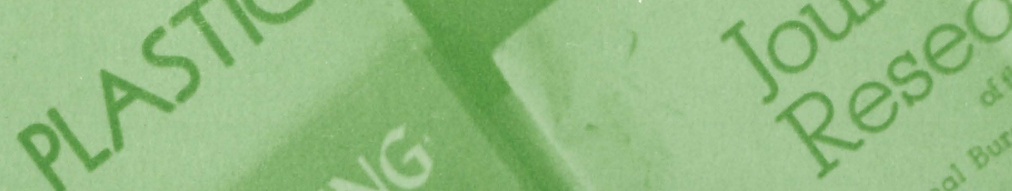 \\ TRHE UNIVERSITY OF MISSQURI ENGINEERING REPRINT-SERIES Reprint Number $>9$
}

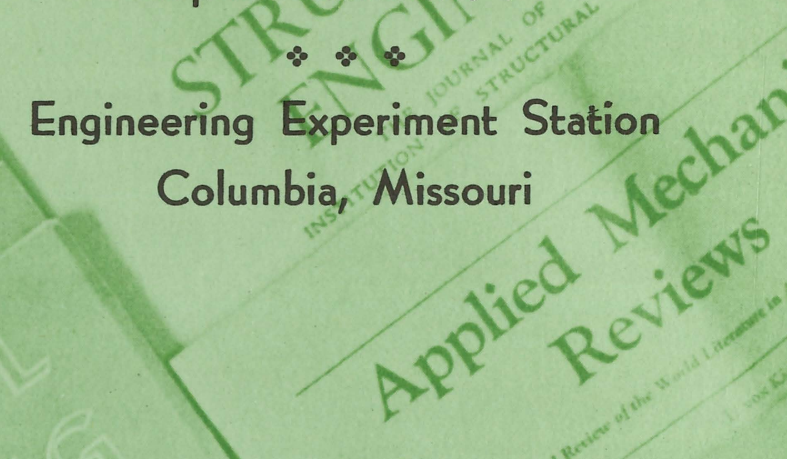

as

A SIATISTICAL DEFINITION OF PERFECT.

MIXTURES OF SOLIDS OF DIEEERENT SIZES

B.

(1) Jüun Sup Hyun, Graduate Student in Chemical Engineering $\sqrt{2}$ University of Missouri

(2) L. E. Marc De Chazal, Professor of Nuclear and Chemical Engineering

2

(S) Reprinted from University of Missouri

$J$ EC Rrocess Design and Development

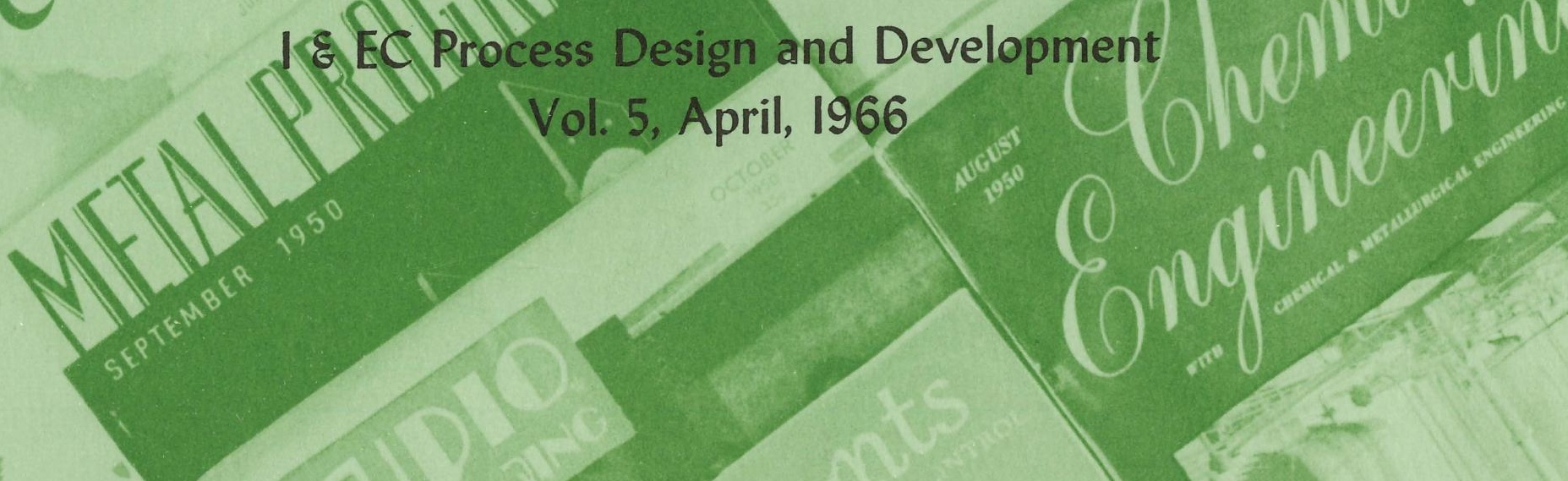




\section{COLLEGE OF ENGINEERING \\ THE ENGINEERING EXPERIMENT STATION}

The Engineering Experiment Station was organized in 1909 as a part of the College of Engineering. The staff of the Station includes all members of the Faculty of the College of Engineering, together with Research Assistants supported by the Station Funds.

The Station is primarily an engineering research institution engaged in the investigation of fundamental engineering problems of general interest, in the improvement of engineering design, and in the development of new industrial processes.

The Station desires particularly to co-operate with industries of Missouri in the solution of such problems. For this purpose, there is available not only the special equipment belonging to the Station but all of the equipment and facilities of the College of Engineering not in immediate use for class instruction.

Inquiries regarding these matters should be addressed to:

The Director

Engineering Experiment Station

University of Missouri

Columbia, Missouri 


\title{
A STATISTICAL DEFINITION OF PERFECT MIXTURES OF SOLIDS OF DIFFERENT SIZES
}

\author{
KUN SUP HYUN ANDL. E. MARC DE CHAZAL \\ Department of Chemical Engineering, University of Missouri, Columbia, Mo.
}

\begin{abstract}
When mixing solids, it is desirable to be able to evaluate the performance of a mixer in terms of its ability to produce mixtures that approach the theoretical limit of uniformity. Although a satisfactory correlation exists for mixtures where the particles are of uniform size, none has yet been developed for multisized particles. A mathematical definition of a perfect mixture of three sizes of particles is proposed. The principles employed can be extended to any number of sizes, but the complexity of the equations increases rapidly. Agreement with experimental results appears substantially better than that obtained by previous investigators.
\end{abstract}

$T$ WO MODELS have been used in attempting to define perfect mixtures of solids. Manning (9), Lacey (8), Buslik (4), Stange (13), and others $(10,14)$ have used the statistical approach, defining mixing in terms of the standard deviation of the composition of samples. Hemelrijk (7) and Blumberg and Maritz (2) used the chi-square distribution for the statistical analysis, but otherwise followed essentially the same approach. On the other hand, Brothman, Wollan, and Feldman (3) and Coulson and Maitra (5) used a kinetic approach based on shear mixing process, which they described as three-dimensional shuffling. Oyama (11) attempted a third approach, and defined as a criterion the specific volume of the mixture of two different sizes of particles. This criterion, however, is demonstrably unreliable since packing arrangement rather than particle distribution is important.

The most common definition of a complete mixture is that which might be obtained by distributing its component particles with equal probability of occupying any position throughout the system. This form of mixing may sometimes produce very poor results in that the mixture is far from homogeneous. Hence, it may be more satisfactory to apply the term complete to the mixing process itself rather than to the resulting mixture. This makes an allowance for the fact that a complete mixing process can still produce bad mixtures. For this reason it has been proposed to replace complete mixing by the term random, which describes the situation more realistically, and we use the term random mixing process instead of mixing. Buslik, Blumberg and Maritz, and Hemelrijk based their statistical consideration on the following definition (7): "A mixing process is called random if all particles are distributed independently in the mixture in such a way that, for every component of the mixture, the probability of finding a particle of this component at a given point is the same for all points in the mixture." The above statement will serve to define our use of the term random, either when used in referring to a random mixture or when speaking of a random distribution.

If a mixture is composed of particles of a single size, Lacey (8) and others $(4,9)$ showed that if the mixture is random, the standard deviation of the composition of samples is equal to the standard deviation that is obtained by applying the binomial distribution:

$$
\sigma=\sqrt{\frac{P(1-P)}{n}}=\operatorname{Lim}_{N \rightarrow \infty} \sqrt{\frac{\Sigma_{i}\left(x_{i}-x\right)^{2}}{N}}
$$

where $n=$ the number of particles in a sample, $x=$ over-all particle fraction of one component in a mixture, $x_{i}=$ particle fraction of one component in the $i$ th sample, $P=$ the ratio of the number of particles of a component to the total number of particles in the mixture, and $N$ is defined as the number of samples analyzed.

Mixtures of nonuniform sizes of particles, however, proved less tractable to theoretical definition. Manning (9) attempted to use his equation for multisized particles, but the relation does not agree well with experimental results. Manning used Equation 1 in the form of

$$
\sigma=\sqrt{\frac{S(1-S) w}{W}}
$$

where $S$ is the weight fraction of large particles, $w$ is the weight of a particle, and $W$ is the weight of each sample. Manning used this equation for the large particles. For mixtures of large and medium particles, he used the following:

$$
\sigma_{y}^{2}=\sigma_{x}^{2}-\sigma_{x+y^{2}}
$$

where $\sigma_{x}^{2}$ and $\sigma_{y}{ }^{2}$ are the variances of large and medium particles, respectively, and $\sigma_{x+y}{ }^{2}$ is the variance of mixtures of large and medium particles.

Buslik modified Manning's model as follows: He assumed every component to be distributed binomially, and he divided the whole mixture into equal subvolumes which he called units. According to the derivation of his equation, each size is binomially distributed throughout the volume of the mixture, with a variance given by:

$$
\sigma_{a}^{2}=\frac{A(1-A) w_{a}+A^{2}\left(w-w_{a}\right)}{W}
$$

where $w_{a}$ is the average particle weight in the size fraction being considered (constant density is assumed), $\bar{w}$ is the average particle weight in the whole mixture, calculated from $\bar{w}=$ $A w_{a}+B w_{b}+C w_{c}$, etc., and $A, B, C$, etc., are the fractions by weight of particle sizes $w_{a}, w_{b}, w_{c}$, etc. $W$ is the total sample weight, and $A$ is the weight fraction being considered.

Although Buslik's model, as expressed in Equation 4 was a substantial improvement on Manning's and gave good results for the large size particle, it was in poor agreement for the smaller sizes.

The failure of Buslik's model appears to stem from an important omission - that is, he does not consider the existence of gaps between large particles in which only small particles can fit. If several sizes of particles are mixed, and if the largest size is randomly distributed, it follows that some of the large particles will be so close together that medium-sized particles cannot fit between them. This volume is not avail- 
able to the medium particles, and the volume in which the latter can be randomly mixed is correspondingly smaller. This error is corrected in the model proposed below. Although the proposed method may be extended to any number of particle sizes, its complexity increases rapidly. Hence, it is presented here for only three sizes of particles.

If we consider a mixture that has $X, Y$, and $Z$ volume fractions of large, medium, and small particles, respectively, the model proposes that, because of their greater mass, the large particles will be unaffected by the smaller particle sizes, except that the latter will provide support. Then, as with Buslik's model, the variance of the large particle content in equal-sized samples is

$$
\sigma_{x}^{2}=\sum_{i=1}^{\infty} P_{i}\left(x-x_{i}\right)^{2} \cong \frac{x(1-x)}{a}
$$

where

$x=$ volume fraction of large particles in the mixture

$x_{i}=$ volume fraction of large particles in the $i$ th sample

$P_{i}=$ probability of having a volume fraction $X_{i}$ in the sample

$a=$ the ratio of the sample volume to the volume of one large particle

Unless one can cut through particles in the sampling process, the number of particles in a sample is an integer, and hence the $x_{i}^{\prime}$ 's are binomially distributed. Thus, unless the sample volume is large compared with the particle size, the inequality in Equation 5 may be important. The magnitude of this inequality is the subject of another study now in progress.

It is now necessary to determine the amount and probability distribution of the space between large particles in which medium particles will not fit. This space, which we designate as excluded volume, is determined below for a simplified model.

Consider a mixture of spherical particles in a spherical sample volume. When two large particles are so close that a medium one cannot fit between them, we may approximate the excluded volume as a cylinder of length and radius $\left(h-r_{m}\right)$ as shown in Figure 1. If we choose to keep one of the large particles fixed in space and allow the other to occupy all positions which will produce a nonzero excluded volume, then the excluded volume is given by the equation

$$
V_{\mathrm{ex}_{1}}=\Pi\left(h-r_{m}\right)^{2} l, \quad 0<l<2 r_{m}
$$

where

$h=\sqrt{\left(r_{l}+r_{m}\right)^{2}-\left(r_{l}+\frac{l}{2}\right)^{2}}=\frac{1}{2} \sqrt{\alpha-\beta l-l^{2}}$ in which we define $\alpha, \beta$, and $\gamma$ as follows:

$$
\begin{aligned}
& \alpha=8 r_{m} r_{l}+4 r_{m}{ }^{2} \\
& \beta=4 r_{l} \\
& \gamma=2 r_{m}
\end{aligned}
$$

In the above set of equations, $l$ is a variable that has meaning for any value between zero and $2 r_{m}$. The probability that $l$ be of a given length is not the same for all lengths. To determine the probability distribution $f(l)$, one must go back to the original premise that one large particle is kept fixed, and that the other is allowed to occupy, with equal probability, all positions which will produce a nonzero volume. Then, since the probability that the centers of the two large particles be a distance $R$ apart is proportional to $R^{2}$, one may see that the distribution function $f(l)$ is expressed by

$$
\int_{0}^{2 r_{m}} f(l) \mathrm{d} l=\int_{0}^{2 r_{m}} \frac{4 \pi\left(2 r_{l}+l\right)^{2} \mathrm{~d} l}{\bar{V}_{v}}=1,0 \leqslant l \leqslant 2 r_{m}
$$

where the range of definition has been limited to values of in which excluded volumes occur, and

$$
\bar{V}_{v}=\frac{4}{3} \pi\left(2 r_{l}+2 r_{m}\right)^{3}
$$

hence,

$$
f(l)=\frac{4 \pi}{\bar{V}_{v}}\left(2 r_{l}+l\right)^{2}, \quad 0 \leqslant l \leqslant 2 r_{m}
$$

The expected volume excluded to medium particles between two large particles is thus

$$
E\left(V_{\mathrm{ex}}\right)_{1}=\int_{0}^{2 r_{m}} V_{\mathrm{ex}_{1}} f(l) \mathrm{d} l
$$

Substituting Equations 6, 7, and 10 into Equation 11, one gets

$$
\begin{aligned}
E\left(V_{\mathrm{ex}}\right)_{1}=\frac{\pi^{2}}{\bar{V}_{v}} \int_{0}^{\gamma}[ & \left(\alpha+\gamma^{2}\right) l-\beta l^{2}-l^{3}-2 \gamma l \times \\
& \left.\sqrt{\alpha-\beta l-l^{2}}\right]\left(\frac{\beta^{2}}{4}+\beta l+l^{2}\right) \mathrm{d} l
\end{aligned}
$$

By integrating and rearranging, we get

$$
\begin{aligned}
& E\left(V_{\text {ex }}\right)_{1}=\frac{\pi^{2}}{\bar{V}_{v}}\left[\left(21.33 r_{m}^{6}+74.97 r_{m}{ }^{5} r_{l}+69.33 r_{m}{ }^{4} r_{l}{ }^{2}+\right.\right. \\
& \left.21.33 r_{m}^{3} r_{l}^{3}\right)-2 \sqrt{\alpha}\left(4.27 r_{m}^{5}+17.07 r_{m}^{4} r_{l}+23.47 r_{m}{ }^{3} r_{l}{ }^{2}+\right. \\
& \left.\left.13.3 r_{m}^{2} r_{l}^{3}-24 r_{m} r_{l}^{4}\right)+16 r_{m} r_{l}\left(r_{m}+r_{l}\right)^{2}\left(\frac{\pi}{2}-\sin ^{-1} \frac{r_{l}}{r_{m}+r_{l}}\right)\right]
\end{aligned}
$$

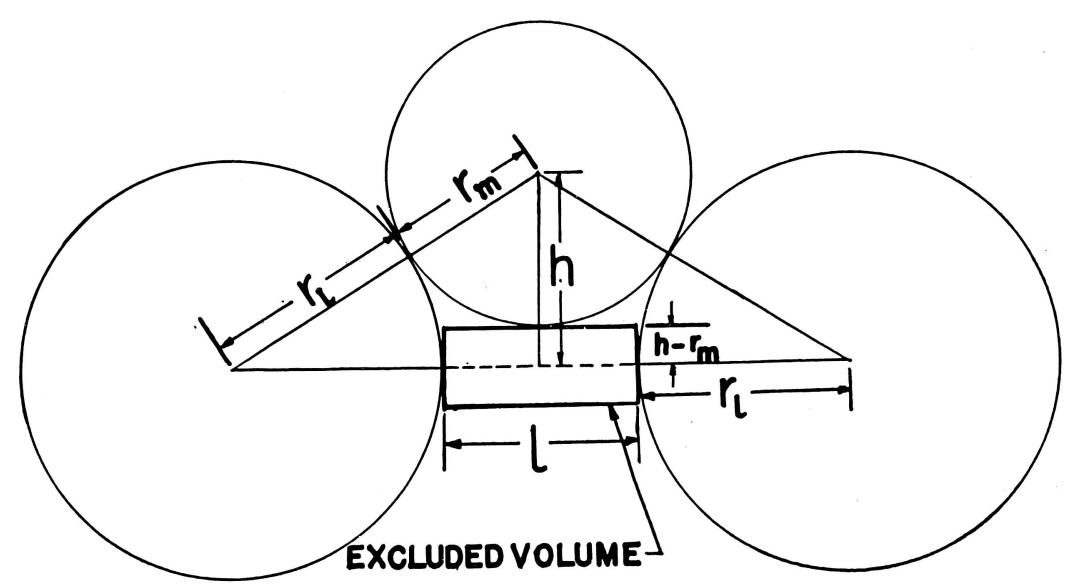

Figure 1. Geometrical model for excluded volume between two large particles 
When we consider more than two particles, there are several possible arrangements that yield excluded volumes. We may consider the geometrical arrangements giving excluded volumes between three particles to fall into two distinct types: 1. Three particles are placed so that the space between each of the three pairs contains an excluded volume. 2. Two particles are close enough to produce an excluded volume, while the third is so placed as to have an excluded volume with either of the other two or with neither, but not with both.

Arrangements of Type 2 are considered two-particle interactions. When considering the excluded volume produced by Type 1 interactions, it is necessary to account only for those excluded volumes which have not been previously accounted for as two-particle interactions. For example, if three large particles, A, B, and $\mathrm{C}$, interact to form excluded volumes between $\mathrm{A}$ and $\mathrm{B}$, between $\mathrm{A}$ and $\mathrm{C}$, and between $\mathrm{B}$ and $\mathrm{C}$, the interactions of $\mathrm{A}$ with $\mathrm{B}$ and of $\mathrm{B}$ with $\mathrm{C}$ are accounted for as Type 2 interactions.

The Type 1 interaction in a three-particle group out of a total of $m$ large particles is the interaction between particles $\mathrm{A}$ and C. Let $E\left(V_{\mathrm{ex}}\right)_{\mathrm{A} / \mathrm{B} \leftrightarrow \mathrm{C}}$ denote the expected excluded volume resulting from this interaction. The ratio of $E\left(V_{\text {ex }}\right)_{\mathrm{A} / \mathrm{B} \leftrightarrow \mathrm{C}}$ to the expected excluded volume between particles $\mathrm{A}$ and $\mathrm{B}$-i.e., $E\left(V_{\mathrm{ex}}\right)_{1}$ may be expressed as the ratio of probabilities of the conditions that the excluded volumes might occur. That is

$$
\frac{E\left(V_{\mathrm{ex}}\right)_{\mathrm{A} / \mathrm{B} \leftrightarrow \mathrm{C}}}{E\left(V_{\mathrm{ex}}\right)_{1}}=\frac{\operatorname{Pr}\left[l_{2}<d_{m} / l_{1}<d_{m}\right]}{\operatorname{Pr}\left[l_{1}<d_{m}\right]}
$$

where $l_{1}=$ the distance between particles $\mathrm{A}$ and $\mathrm{B}$

$l_{2}=$ the distance between particles $\mathrm{A}$ and $\mathrm{C}$

On the other hand, the probability that particle $\mathrm{C}$ is so located as to produce an excluded volume between $\mathrm{A}$ and $\mathrm{C}$ is a function of the volume around $\mathrm{A}$ which is not occupied by other particles. Let $a_{2}$ be the ratio of the probability that $l_{2}$ is less than $d_{m}$ when $l_{1}$ is also less than $d_{m}$ divided by the probability that $l_{1}$ is less than $d_{m}$, then

$$
\begin{aligned}
a_{2}=\frac{\operatorname{Pr}\left[l_{2}<d_{m} / l_{1}<d_{m}\right]}{\operatorname{Pr}\left[l_{1}<d_{m}\right]} & = \\
& \frac{\left[4 \pi\left(d_{l}+d_{m}\right)^{2}-\frac{\pi}{4} d_{l}{ }^{2}\right](m-2)}{\left[4 \pi\left(d_{l}+d_{m}\right)^{2}(m-1)\right.}
\end{aligned}
$$

Combining 14 and 15 one can write

$$
E\left(V_{\mathrm{ex}}\right)_{\mathrm{A} / \mathrm{B} \leftrightarrow \mathrm{C}}=a_{2} E\left(V_{\mathrm{ex}}\right)_{1}
$$

Therefore, the total expected excluded volume caused by particle $\mathrm{A}$ in a three-particle group is expressed as $E\left(V_{\text {ex }}\right)_{2}$ and has the value

$$
E\left(V_{\mathrm{ex}}\right)_{2}=E\left(V_{\mathrm{ex}}\right)_{1}\left(1+a_{2}\right)
$$

Similarly, if four particles, A, B, C, and D, are involved, the contribution of one particle is:

$$
\begin{aligned}
E\left(V_{\mathrm{ex}}\right)_{3} & =E\left(V_{\mathrm{ex}}\right)_{2}+a_{3} E\left(V_{\mathrm{ex}}\right)_{1} \\
& =E\left(V_{\mathrm{ex}}\right)_{1}\left(1+a_{2}+a_{3}\right)
\end{aligned}
$$

where

$$
\begin{aligned}
a_{3}=\frac{\operatorname{Pr}\left[l_{3}<d_{m} / l_{2}<d_{m}, l_{1}<d_{m}\right]}{\operatorname{Pr}\left[l_{1}<d_{m}\right]} & = \\
& \frac{(m-3)\left[4 \pi\left(d_{l}+d_{m}\right)^{2}-\frac{2 \pi}{4} d_{l}^{2}\right]}{(m-1)\left[4 \pi\left(d_{l}+d_{m}\right)^{2}\right]}
\end{aligned}
$$

and $l_{3}=$ the distance between particle $\mathrm{A}$ and $\mathrm{D}$.
By extension the interaction of one particle and $n$ others will produce an expected excluded volume about that particle equal to

$$
E\left(V_{\mathrm{ex}}\right)_{n}=E\left(V_{\mathrm{ex}}\right)_{1}\left(1+a_{2}+a_{3} \ldots+a_{n}\right)
$$

where

$$
\begin{aligned}
a_{n}=\frac{\operatorname{Pr}\left[l_{n}<d_{m} / l_{n-1}<d_{m}, \ldots, l_{1}<d_{m}\right]}{\operatorname{Pr}\left[l_{1}<d_{m}\right]}= \\
\frac{(m-n)\left[4 \pi\left(d_{l}+d_{m}\right)^{2}-\frac{(n-1)}{4} \pi d_{l}^{2}\right]}{(m-1)\left[4 \pi\left(d_{l}+d_{m}\right)^{2}\right]}
\end{aligned}
$$

Let $\bar{V}_{\text {ex }}=E\left(V_{\text {ex }}\right)_{n}$, the expected excluded volume between one particle and $n$ others. After simplification of the expression for $a_{1}$ through $a_{n}$ and substitution into Equation 20,

$$
\begin{gathered}
\bar{V}_{\mathrm{ex}}=E\left(V_{\mathrm{ex}}\right)_{1}\left[\left(1+\frac{m-2}{m-1}+\ldots+\frac{m-n}{m-1}\right)-\right. \\
\left.\frac{d_{l}^{2}}{16\left(d_{l}+d_{m}\right)^{2}} \frac{(m-2)+2(m-3)+\ldots+(n-1)(m-n)}{(m-1)}\right] \\
=E\left(V_{\mathrm{ex}}\right)_{1}\left[\frac{n(2 m-n-1)}{2(m-1)}-\frac{d_{l}{ }^{2}}{96\left(d_{l}+d_{m}\right)^{2}} \times\right. \\
\left.\frac{m(n-1)(3 m-2 n-2)}{(m-1)}\right]
\end{gathered}
$$

The maximum number, $n$, of particles that can interact with a central one is readily seen to be given approximately by

$$
n_{\max }=\frac{4 \pi\left(d_{l}+d_{m}\right)^{2}}{\frac{\pi}{4} d_{l}^{2}}=16\left(1+\frac{d_{m}}{d_{l}}\right)^{2}
$$

It is now possible to simplify Equation 22 by substituting in Equation 23 for the value of $n$. If we further substitute $d d m /{ }_{l}=\omega$, Equation 22 becomes

$$
\begin{aligned}
\bar{V}_{\text {ex }}= & E\left(V_{\text {ex }}\right)_{1}\left[\frac{m}{m-1}\left(8.5+16 \omega+8 \omega^{2}\right)-\frac{1}{m-1} \times\right. \\
& \left.\left(52.6+186.7 \omega+264 \omega^{2}+170.6 \omega^{3}+42.6 \omega^{4}\right)\right]
\end{aligned}
$$

For the sample containing $m$ large particles, the total expected excluded volume is readily seen to be the sum of the expected excluded volumes about each particle less those parts which are already accounted for about another particle. The total expected excluded volume thus is

$$
\left(V_{\mathrm{ex}}\right)_{m}=\frac{m-1}{m} \bar{V}_{\mathrm{ex}}+\frac{m-2}{m} \bar{V}_{\mathrm{ex}}+\ldots+\frac{1}{m} \bar{V}_{\mathrm{ex}}=\frac{m-1}{2} \bar{V}_{\mathrm{ex}}
$$

Substituting Equation 24 into Equation 25, we get

$$
\begin{aligned}
\left(V_{\mathrm{ex}}\right)_{m}= & E\left(V_{\mathrm{ex}}\right)_{1}\left[m\left(4.25+8 \omega+4 \omega^{2}\right)-\right. \\
& \left.\left(26.3+93.3 \omega+132 \omega^{2}+85.3 \omega^{3}+21.3 \omega^{4}\right)\right]
\end{aligned}
$$

Up to this point, we have dealt with a sample containing $m$ particles. Since $m$ is not a constant in the general case, the expected excluded volume for a sample of volume $V_{\mathrm{sa}}$ becomes

$$
\begin{aligned}
E\left(V_{\mathrm{ex}}\right)= & E\left(V_{\mathrm{ex}_{1}}\right)\left[\left(4.25+8 \omega+4 \omega^{2}\right) E(m)-\right. \\
& \left.\left(26.3+93.3 \omega+132 \omega^{2}+85.3 \omega^{3}+21.3 \omega^{4}\right)\right]
\end{aligned}
$$


where $E(m)$ is the expected number of large particles in the sample of size $V_{\text {sa }}$ :

$$
E(m)=\frac{V_{\mathrm{sa}} x}{V_{l}}
$$

Since $V_{\mathrm{sa}}$ and $V_{l}$ are constants,

$$
\sigma_{m}^{2}=\left(\frac{V_{\mathrm{sa}}}{V_{l}}\right)^{2} \sigma_{x}^{2}
$$

For a given mixture, $E\left(V_{\mathrm{ex}}\right)_{1}$ is a constant; thus, the variance of the excluded volume fraction can be obtained from Equations 27, 28, and 29 as

$$
\sigma_{\mathrm{ex}}^{2}=\left[\frac{E\left(V_{\mathrm{ex}}\right)_{1}}{V_{l}}\left(4.25+8 \omega+4 \omega^{2}\right)\right]^{2} \sigma_{x}{ }^{2}
$$

One must now consider the distribution of medium and small particles in the space remaining after the large particles have been fixed in space.

The total sample volume can be divided into three distinct subvolumes: $V_{l}$, the volume occupied by the large particles; $V_{\text {ex }}$, the excluded volume in which only small particles may fit; and $V_{m+s}$, the volume available to medium and small particles alike.

For a random mixture, the expected values of the subvolumes are

$$
\begin{aligned}
& E\left(V_{l}\right)=X . V_{\mathrm{sa}} \\
& E\left(V_{\mathrm{ex}}\right)=E\left(V_{\mathrm{ex}}\right)_{1}\left[\left(4.25+8 \omega+4 \omega^{2}\right) \frac{V_{\mathrm{sa}} X}{V_{l}}-\right. \\
& \left.\quad\left(26.3+93.3 \omega+132 \omega^{2}+85.3 \omega^{3}+21.3 \omega^{4}\right)\right]
\end{aligned}
$$

$E\left(V_{m+s}\right)=E\left(V_{\mathrm{sa}}\right)-E\left(V_{l}\right)-E\left(V_{\mathrm{ex}}\right)=V_{\mathrm{sa}}(1-X)-E\left(V_{\mathrm{ex}}\right)$

The expected standard deviations of the volume fractions occupied by the medium and small particles are found in a manner similar to that for the large particles. The volume in which they can be found is less than the total volume by that occupied by the large particles and by the excluded volume, so that the expected concentration of medium particles must be increased, and the variance becomes

$$
\sigma_{y}^{2}=\frac{y^{\prime}\left(1-y^{\prime}\right)}{b}
$$

where

$$
y^{\prime}=\frac{V_{a s}}{E\left(V_{m+s}\right)} \quad y, \quad b=\frac{E\left(V_{m+s}\right)}{V_{m}}
$$

and $y=$ fraction of medium particles in the mixture.

For the small particles present in $V_{m+s}$ the standard deviation is constrained to the same value as $\sigma_{y}$. By definition, the excluded volume $V_{\mathrm{ex}}$, consists of pockets of unmixed small particles. The total volume occupied by small particles is the excluded volume plus the volumes occupied by those in $V_{m+s}$. Let us denote $V_{\mathrm{sm}}$ as the volume of small particles in $V_{m+s}$. Hence, $V_{s}=V_{\mathrm{sm}}+V_{\mathrm{ex}}$, with variance

$$
\sigma_{z}^{2}=\sigma_{z}^{2}(y)+\sigma_{z}^{2}(\mathrm{ex})
$$

where $\sigma_{z}^{2}(y)=$ contribution to the small particle distribution variance from volumes where medium size particles are present

$\sigma_{z}^{2}(\mathrm{ex})=$ contribution to the small particle distribution variance from the excluded volumes

But, since

$$
\sigma_{z}^{2}(y)=\sigma_{y}^{2}
$$

and

Equation 33 becomes

$$
\sigma_{z}^{2}(\mathrm{ex})=\sigma_{\mathrm{ex}}^{2}
$$

$$
\sigma_{z}^{2}=\sigma_{y}^{2}+\sigma_{\mathrm{ex}}^{2}
$$

The expected volumes and associated values of the variances for a "random" mixture are presented in Table I. Experimentally, the expected variances can be obtained from the equations

$$
\begin{gathered}
\hat{\sigma}_{x}^{2}=\frac{1}{N(N-1)} \sum\left(x_{i}-\bar{x}\right)^{2}+\frac{1}{N-1}(x-\bar{x})^{2} \\
\hat{\sigma}_{y}^{2}=\frac{1}{N(N-1)} \sum\left(y_{i}-\bar{y}\right)^{2}+\frac{1}{N-1}(y-\bar{y})^{2} \\
\hat{\sigma}_{z}^{2}=\frac{1}{N(N-1)} \sum\left(Z_{i}-\bar{Z}\right)^{2}+\frac{1}{N-1}(Z-\bar{Z})^{2}
\end{gathered}
$$

The extent of the agreement between the experimental values obtained for Equations 35, 36, and 37, and the values of the variances obtained from Equations 5, 32, and 34, respectively, should give weight to the validity of the authors' model. Some testing was done, as described below.

Table I. Expected Volumes and Variances in a Fully Mixed Mixture of Three Different Sizes, as Derived in This Paper
Particle

\begin{tabular}{|c|c|c|c|c|c|c|}
\hline \multirow[b]{2}{*}{$\begin{array}{l}\text { Run } \\
\text { No. }\end{array}$} & \multirow[b]{2}{*}{ Size } & \multicolumn{2}{|r|}{$E\left(V_{\mathrm{ex}}\right)$} & \multicolumn{3}{|c|}{ Standard Deviation, $\%$} \\
\hline & & $\begin{array}{c}\text { Av. } \\
\text { Fraction, } \\
\%\end{array}$ & $\begin{array}{c}E\left(V_{m+s}\right)+ \\
E\left(V_{\text {ex }}\right), \\
\%\end{array}$ & $\begin{array}{c}\text { By } \\
\text { Buslik's } \\
\text { model }\end{array}$ & $\begin{array}{c}\text { By } \\
\text { Eqns. 5, } \\
30,32\end{array}$ & $\begin{array}{l}\text { From } \\
\text { exptl. } \\
\text { data }\end{array}$ \\
\hline 1 & $\begin{array}{l}\mathrm{L} \\
\mathrm{M} \\
\mathrm{S}\end{array}$ & $\begin{array}{l}33.0 \\
32.5 \\
34.5\end{array}$ & 29.12 & $\begin{array}{l}0.986 \\
0.690 \\
0.654\end{array}$ & $\begin{array}{l}1.145 \\
0.7972 \\
0.7976\end{array}$ & $\begin{array}{l}1.187 \\
0.735 \\
0.786\end{array}$ \\
\hline $2 \mathrm{a}$ & $\begin{array}{l}\mathrm{L} \\
\mathrm{M} \\
\mathrm{S}\end{array}$ & $\begin{array}{l}33.3 \\
33.5 \\
33.2\end{array}$ & 28.36 & $\begin{array}{l}0.942 \\
0.657 \\
0.623\end{array}$ & $\begin{array}{l}1.096 \\
0.7665 \\
0.7669\end{array}$ & $\begin{array}{l}0.980 \\
0.750 \\
0.820\end{array}$ \\
\hline $2 \mathrm{~b}$ & $\begin{array}{l}\mathrm{L} \\
\mathrm{M} \\
\mathrm{S}\end{array}$ & $\begin{array}{l}33.6 \\
33.6 \\
32.8\end{array}$ & 26.38 & $\begin{array}{l}0.944 \\
0.659 \\
0.625\end{array}$ & $\begin{array}{l}1.100 \\
0.7690 \\
0.7694\end{array}$ & $\begin{array}{l}1.028 \\
0.762 \\
0.870\end{array}$ \\
\hline 3 & $\begin{array}{l}\mathrm{L} \\
\mathrm{M} \\
\mathrm{S}\end{array}$ & $\begin{array}{l}33.1 \\
33.9 \\
33.0\end{array}$ & 26.39 & $\begin{array}{l}0.939 \\
0.650 \\
0.617\end{array}$ & $\begin{array}{l}1.092 \\
0.7597 \\
0.7600\end{array}$ & $\begin{array}{l}1.093 \\
0.600 \\
0.883\end{array}$ \\
\hline
\end{tabular}
Size

\section{Expected

\begin{tabular}{|c|c|c|c|c|}
\hline \multirow[b]{2}{*}{$\begin{array}{c}\text { Particle } \\
\text { Size }\end{array}$} & \multirow[b]{2}{*}{$\begin{array}{c}\text { Av. } \\
\text { Fraction, } \\
\%,\end{array}$} & \multicolumn{2}{|l|}{ Mixing } & ation \\
\hline & & $\begin{array}{c}E\left(V_{m+s}\right)+ \\
E\left(V_{\text {ex }}\right) \\
\%,\end{array}$ & $\begin{array}{l}\text { By Eqns. } \\
5,30,32\end{array}$ & $\begin{array}{l}\text { From } \\
\text { exptl. } \\
\text { data }\end{array}$ \\
\hline $\begin{array}{l}-24+28 \\
-32+35 \\
-42+48\end{array}$ & $\begin{array}{l}32.6 \\
33.7 \\
33.7\end{array}$ & 9.61 & $\begin{array}{l}0.682 \\
0.543 \\
0.543\end{array}$ & $\begin{array}{l}0.704 \\
0.226 \\
0.797\end{array}$ \\
\hline
\end{tabular} Volume}
Variance of Volume Fraction
$(x)\left(V_{\mathrm{sa}}\right) \quad \sigma_{x}^{2}=\frac{x(1-x)}{a} ; a=V_{\mathrm{s}} / v_{1}$
$(y)\left(V_{\mathrm{sa}}\right) \quad \sigma_{y}{ }^{2}=\frac{y^{\prime}\left(1-y^{\prime}\right)}{b} ; b=E\left(V_{m+s}\right) / v_{m}$
$y^{\prime}=V_{\mathrm{sa}} / E\left(V_{m+s}\right) y$
$(z)\left(V_{\mathrm{sa}}\right) \quad \sigma_{x}^{2}=\sigma_{y}^{2}+\sigma_{\mathrm{ex}^{2}}$

Large

Medium

Small

Excluded volume $E\left(V_{\mathrm{ex}}\right) \quad \sigma_{\mathrm{ex}}{ }^{2}=\left[\frac{E\left(V_{\mathrm{ex}}\right)_{1}}{V_{l}}(4.25+8 \omega+\right.$

$$
\left.\left.4 \omega^{2}\right)\right]^{2} \sigma_{x}^{2}
$$

Table II. Calculation of Standard Deviation in Silica Gel Mixing

Table III. Calculation of Standard Deviation in Glass Beads 


\section{Experimental Equipment and Procedure}

To test the mathematical model, graded particles were mixed in a drum mixer. The drum was made from a 9-inch length of standard 8-inch steel pipe, internally machined to a diameter of 8 inches, and split longitudinally into six identical pieces. The end plates were machined from mild steel and fastened to $5 / 8$-inch drive shafts by means of an aluminum collar bolted to the plates. The shafts were supported by sleeve bearings placed on a wooden box.

The mixer was driven by a motor and pulley arrangement to produce a speed of 36 r.p.m. Thin steel flights were placed longitudinally in the mixer to improve mixing.

Two types of particles were used for mixing-glass beads of screen fractions $(-24+28),(-32+35)$, and $(-42+48)$; and silica gel of screen fractions $(-14+16),(-20+24)$, and $(-28+32)$.

As a starting condition, the drum was divided into three separate compartments by two vertical partitions parallel to the axis of the drum. For both types of mixes, equal volumes of the three different sizes were placed in the mixer, one in each of the compartments. The partitions were carefully removed after the surface was levelled, and the mixer was closed. The mixer was then run long enough for mixing to reach equilibrium. This required more than 900 revolutions. The mixer was then stopped, and 20 samples were randomly taken for analysis. Randomness of sampling was ensured by allocating numbers to 100 sectors in the mixture and selecting the sectors to sample by use of a table of random numbers (12).

A plastic scoop was used to collect the samples in each layer, and a vacuum hose was used to remove the remainder of each layer after sampling. A thin layer was then skimmed off before the next set of samples was collected. Each sample was separated into its three component sizes by screening, and the size fractions were separately weighed to the nearest $0.1 \mathrm{mg}$. The experimental standard deviations were then calculated by Equations 35, 36, and 37.

\section{Results and Discussion}

Table II compares the standard deviations calculated from Equations 5, 33, and 34 with the standard deviations by Buslik's formula and the experimental data for three runs using silica gel particles.

In both theoretical and experimental evaluations of standard deviation, uniform density is assumed throughout the mixture, and the packing effect is considered. The packing effect gives the ratio of the volumes before and after packing to be 1.16 .

A series of runs was made using the glass beads with various sizes of flights. The results obtained are shown in Table III for comparison. The differences in theoretical and experimental values of $\sigma_{s}{ }^{\prime}$ in Table II are small while the differences for $\sigma_{y}$ and $\sigma_{z}$ in Table III are much greater. These larger discrepancies in Table III are due to apparent inability to mix smooth spheres completely. In Table III, the estimate of the standard deviation for the medium particles is much less than the theoretical, and for the small particles is substantially more than the theoretical value. This fact was characteristic of the bad mixtures made, whether with glass beads or silica gel, and is perhaps an index of poor mixing far more sensitive than the estimated large particle standard deviation.

The equations developed in this paper agree with the experimental results significantly better than do those from Buslik's model. Analysis using the F-test $(1,6)$ shows no significant difference between the experimental results for the silica gel mixes and the calculated values. On the other hand, Buslik's calculated variance for the small size particles was different from the experimental results with $98 \%$ confidence. The Ftest on the two models showed significantly different variances for all particle sizes.

The method above was presented for three sizes of particles. To extend the method to more than three sizes, one must consider the excluded volumes between all particles larger than the one under consideration. Thus, if one were to consider particles of size 4, where particles of size 1, 2, and 3 are larger, the interactions that result in excluded volumes are between $1-1,1-2,1-3,2-2,2-3$, and $3-3$. If the mixture under study contains $n$ distinct sizes, the total number of contributions to the excluded volumes will be:

$$
\sum_{j=1}^{n-2}\left(\sum_{i=0}^{j} i\right)
$$

\section{Acknowledgment}

The problem of defining a mixture of multi-sized solids was brought to the attention of one of the authors while working in the Chemical Engineering Division of the Atomic Energy Research Establishment, Harwell, England. The discussions with P. M. Lacey were particularly helpful in bringing out the main difficulties involved.

\section{Nomenclature}

\begin{tabular}{|c|c|}
\hline$A, B, C$ & $=$ weight fractions \\
\hline & $=V_{\mathrm{sa}} / V_{l}$ \\
\hline $\begin{array}{l}a_{2}, a_{3}, \ldots a_{n} \\
b\end{array}$ & $\begin{array}{l}=\text { correction factors } \\
=E\left(V_{m+s}\right) / v_{m}\end{array}$ \\
\hline$d_{l}, d_{m}$ & $\begin{array}{l}=\text { diameter of a large and medium particle, } \\
\text { respectively }\end{array}$ \\
\hline$E\left(V_{\mathrm{ex}}\right)$ & $=$ expected value of $V_{\mathrm{ex}}$ \\
\hline$E\left(V_{\text {ex }}\right)_{n}$ & $\begin{array}{l}=\text { expected value of the excluded volume be- } \\
\text { tween }(n-1) \text { large particles and one } \\
\text { fixed large particle }\end{array}$ \\
\hline$E\left(V_{m+s}\right)$ & $=$ expected value of $V_{m+s}$ \\
\hline & y density function of $l$ \\
\hline$F(l)$ & $=$ distributi \\
\hline$l$ & $=$ distance between two lar \\
\hline$m$ & $=$ number of large particles \\
\hline$N$ & es analyzed in a mixture \\
\hline$n$ & $=$ number of particles in a sample \\
\hline$n_{\max }$ & $=\underset{\text { central one }}{\text { maximum number of large particles around a }}$ \\
\hline$P$ & of a component in the mixture \\
\hline$P_{i}$ & $=$ binomial distribution probability \\
\hline$r_{l}, r_{m}$ & $\begin{array}{l}=\text { radius of a large and medium particle, re- } \\
\text { spectively }\end{array}$ \\
\hline$s$ & $=$ weight fraction of large particles \\
\hline$V_{\mathrm{ex}}$ & $=$ exclud \\
\hline$V_{l}$ & $=$ volume of large particles \\
\hline$V_{m+s}$ & $\begin{array}{l}=\text { volume available to medium and small par- } \\
\text { ticles alike }\end{array}$ \\
\hline$V_{v}$ & $=$ constant \\
\hline & $=$ sample volume \\
\hline$v_{l}, v_{m}, v_{s}$ & $\begin{array}{c}=\text { volume of a large, medium, and small } \\
\text { particle, respectively }\end{array}$ \\
\hline$W$ & $=$ weight of a sample \\
\hline$w$ & $=$ weight \\
\hline $\bar{w}$ & $=$ average particle weight in th \\
\hline$x, y, z$ & $\begin{array}{l}=\text { volume fraction of large, medium, and small } \\
\text { particles in a mixture, respectively }\end{array}$ \\
\hline $\bar{x}, \bar{y}, \bar{z}$ & $\begin{array}{l}=\text { mean fraction of large, medium, and small } \\
\text { particles in } N \text { samples, respectively }\end{array}$ \\
\hline$x_{i}, y_{i}, z_{i}$ & $\begin{array}{l}=\text { fraction of large, medium, and small par- } \\
\text { ticles in } i \text { th sample }\end{array}$ \\
\hline$y^{\prime}$ & $=$ modified function of medium particles \\
\hline & $=$ standard deviation \\
\hline$\sigma_{\mathrm{ex}}^{2}$ & $=$ variance of the excluded volume fraction \\
\hline$\sigma_{m}^{2}$ & $\begin{array}{l}=\text { variance of a number of large particles in the } \\
\text { samples }\end{array}$ \\
\hline & variance, of large, medium, and small par- \\
\hline
\end{tabular}




\begin{tabular}{|c|c|}
\hline$\sigma_{x+y^{2}}$ & $\begin{array}{c}=\text { variance of large and medium particles } \\
\text { together }\end{array}$ \\
\hline$\sigma_{z}^{2}(\mathrm{ex})$ & $\begin{array}{l}=\text { contribution to variance of small particles } \\
\text { from the excluded volume }\end{array}$ \\
\hline$\sigma_{z}^{2}(y)$ & $\begin{array}{l}=\text { contribution to variance of small particles } \\
\text { from volume where medium size particles } \\
\text { may be present }\end{array}$ \\
\hline$\hat{\sigma}_{x_{l}}{ }^{2}, \hat{\sigma}_{y l}{ }^{2}, \hat{\sigma}_{z}^{2}$ & $\begin{array}{l}=\text { estimated variance of large, medium, and } \\
\text { small particles in the mixture, respec- } \\
\text { tively }\end{array}$ \\
\hline$\omega$ & $=$ the ratio $d m / d_{l}$ \\
\hline
\end{tabular}

Literature Cited

(1) Bennett, G. A., Franklin, N. L., "Statistical Analysis in Chemistry and the Chemical Industry," pp. 319-23, New York, 1954

(2) Blumberg, R., Maritz, J. S., Chem. Eng. Sci. 12, 240 (1953).

(3) Brothman, A., Wollan, G. N., Feldman, S. M., Chem. Met. Eng. 52, 102 (1945).
(4) Buslik, D., ASTM Bull. No. 66, April, 1950.

(5) Coulson, J. M., Maitra, N. K., Ind. Chemist 26, 55 (1950).

(6) Davies, O. L., "Statistical Methods in Research and Produc tion," 3rd ed., pp. 368-71, London, 1958.

(7) Hemelrijk, J., "Statistical Methods Applied to the Mixing of Solid Particles," Math. Centrum Bull. S159, Amsterdam, 1954.

(8) Lacey, P. M. G., Trans. Inst. Chem. Engrs., London 21, 52 (1943).

(9) Manning, S. B., J. Inst. Fuel 56, 153 (1937).

(10) Mayagi, S., J. Ceram. Assoc. Japan 58, 417 (1950).

(11) Oyama, Y., Sci. Papers Inst. Phys. Chem. Res. (Tokyo) 37, 951 (1940).

(12) Rand Corp., "A Million Random Digits with 100,000 Normal Deviates," Free Press, Glencoe, Ill., 1955.

(13) Stange, K., Chem. Eng. Tech. 26, 331 (1954).

(14) Weidenbaum, S. S., Bonilla, C. F., Chem. Eng. Progr. 51, 27-J (1955).

RECEIVED for review July 23, 1962 Resubmitted August 6, 1965 AGGEPTED December 6, 1965
A reprint from

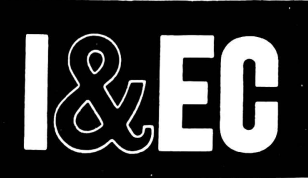

PROCESS DESIGN AND DEVELOPMENT

Vol. 5, April 1966, Pages 105-110

Copyright 1966 by the American Chemical

Society and reprinted by permission of the copyright owner 


\section{PUBLICATIONS OF THE ENGINEERING REPRINT SERIES}

Copies of publications may be secured from the Director of the Engineering Experiment Station, University of Missouri. Single copies may be obtained free unless otherwise indicated until the supply is exhausted. Requests for additional copies will be considered upon further inquiry.

Reprint No.

66. Variable-Mesh Difference Equation for the Stream Function in Axially Symmetric Flow by J. C. Lysen, Associate Professor, Mechanical Engineering, University of Missouri. Reprinted from AIAA Journal, 1964.

67. Creep of Concrete: Influencing Factors and Prediction by A. M. Neville, Chairman, Division of Engineering, University of Alberta, Calgary, and B. L. Meyers, Assistant Professor of Civil Engineering, University of Missouri.

Effect of Creep and Shrinkage on the Behavior of Reinforced Concrete Members by A. Pauw, Professor and Chairman of Civil Engineering, and B. L. Meyers, Assistant Professor of Civil Engineering, University of Missouri. Reprinted from Symposium on Creep on Concrete, Publication SP-9, The American Concrete Institute.

68. A Method of Data List Processing with Application to EEG Analysis by C. M. Philpott, Control Data Corporation, St. Paul, Minnesota, and G. B. Lago, Professor of Electrical Engineering, University of Missouri. Reprinted from Communications of the ACM, Volume 8, Number 5, May, 1965.

69. Method for Obtaining the Trees of a $v$ Vertex Complete Graph from the Trees of a v-1 Vertex Complete Graph by G. W. Zobrist, Assistant Professor of Electrical Engineering, University of Missouri, and G. V. Lago, Professor of Electrical Engineering, University of Missouri. Reprinted from the Matrix and Tensor Quarterly, Volume 15, Number 3, March, 1965.

70. Treatment of Livestock Waste-A Laboratory Study by E. A. Jeffrey, W. C. Blackmann, Jr., and Ralph Ricketts. Reprinted from Transactions of the ASAE, Volume 8, Number 1.

71. The Electronic Position Indicator by Richard P. Covert, Associate Professor of Industrial Engineering, University of Missouri. Reprinted from The Journal of Industrial Engineering, Volume XVI, No. 4, July-August, 1965, pages 255-259.

72. The Reflected Impedance of a Circular Coil in the Proximity of a Semi-Infinite Medium by David H. S. Cheng. Reprinted from IEEE Transactions on Instrumentation and Measurement, Volume IM-14, Number 3, September, 1965.

73. Irrotational Flow Over Spillways of Finite Height by John J. Cassidy, Associate Professor of Civil Engineering, University of Missouri. Reprinted from Journal of the Engineering Mechanics Division, Proceedings of the American Society of Civil Engineers, Volume 91, Number EIM6, December, 1965.

74. Experimental Investigation of a Magnetically Balanced Arc in a Transverse Argon Flow by T. W. Myers, Visiting Research Associate, Thermo-Mechanics Research Laboratory, Wright-Patterson AFB, Ohio, C. N. McKinnon, Instructor of Mechanical Engineering, University of Missouri, and J. C. Lysen, Associate Professor of Mechanical Engineering, University of Missouri. Reprinted from Journal of Engineering for Power, January, 1966.

75. A Study of the Effects of Paced Audio-Rhythm on Repetitive Motion by John A. Conte, Undergraduate Industrial Engineer, University of Missouri. Reprinted from The Journal of Industrial Engineering, * Volume XVII, Number 3, 1966.

76. Measured and Computed Stresses in Three Castellated Beams by Richard T. Douty, Associate Professor of Civil Engineering and James W. Baldwin, Professor of Civil Engineering, University of Missouri. Reprinted from AISC Journal, January, 1966.

77. A Method of Obtaining a Uniform Electric Field by A. V. Dralle and D. L. Waidelich. Reprinted from 1966 IEEE International Convention Record, Part 7.

78. The Use of the Flux Plot in Traffic Control by Robert J. Wheeler, Associate Professor of Civil Engineering and Elmer M. Tory, Associate Professor Mathematics, Mount Allison University. Reprinted from the Traffic Quarterly, July 1965.

79. A Statistical Definition of Perfect Mixtures of Solids of Different Sizes by Kun Sup Hyun, Graduate Student in Chemical Engineering, and L. E. March De Chazal, Professor of Nuclear and Chemical Engineering, University of Missouri. Reprinted from I \& EC Process Design and Development, Vol. 5, April 1966. 
University of Missouri Libraries

University of Missouri

MU Engineering Experiment Station Series

Local Identifier

Hyun 1966

Capture information

Date captured 2018 June

Scanner manufacturer Ricoh

Scanner model

MP C4503

Scanning software

Optical resolution

Color settings

File types

600 dpi

Grayscale, 8 bit; Color 24 bit Tiff

Source information

Format

Content type

Notes
Book

Text

Digitized duplicate copy not retained in collection.

Derivatives - Access copy

Compression

Editing software

Resolution

Color

File types

Notes

\section{LZW}

Adobe Photoshop

600 dpi

Grayscale, 8 bit; Color, 24 bit

Tiffs converted to pdf

Greyscale pages cropped and canvassed. Noise removed from background and text darkened.

Color pages cropped. 